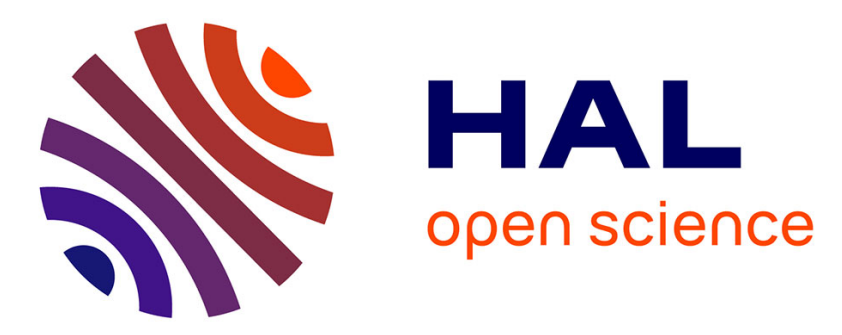

\title{
Analysis of Macro Diversity based on Maximum Ratio Combining in Long Range ALOHA Networks
}

\author{
Qipeng Song, Loutfi Nuaymi, Xavier Lagrange
}

\section{To cite this version:}

Qipeng Song, Loutfi Nuaymi, Xavier Lagrange. Analysis of Macro Diversity based on Maximum Ratio Combining in Long Range ALOHA Networks. Telecommunication Systems, 2019, 10.1007/s11235019-00579-3 . hal-02140290

\section{HAL Id: hal-02140290 https://imt-atlantique.hal.science/hal-02140290}

Submitted on 27 May 2019

HAL is a multi-disciplinary open access archive for the deposit and dissemination of scientific research documents, whether they are published or not. The documents may come from teaching and research institutions in France or abroad, or from public or private research centers.
L'archive ouverte pluridisciplinaire HAL, est destinée au dépôt et à la diffusion de documents scientifiques de niveau recherche, publiés ou non, émanant des établissements d'enseignement et de recherche français ou étrangers, des laboratoires publics ou privés. 


\title{
Analysis of Macro Diversity based on Maximum Ratio Combining in Long Range ALOHA Networks*
}

\author{
Qipeng Song $\quad$ Loutfi Nuaymi Xavier Lagrange ${ }^{\dagger}$
}

27 mai 2019

\begin{abstract}
Résumé
In low power wide area networks (LPWAN), packets transmitted by a device are not addressed to a specific base station : any surrounding station can receive them. This opens the way to several macro diversity schemes. As opposed to independent decoding of packets received by each base station, we propose using maximum ratio combining (MRC) to improve system performance. We use stochastic geometry to analyze the packet loss probability when MRC is implemented for both pure and slotted Aloha and obtain a closed formula when the path loss exponent $\gamma$ is 4 . However, the formula is valid when all base stations on an infinite plane participate in the MRC procedure, which gives the most optimistic evaluation. We developed simulations to get the performance when a finite number of receivers in the MRC is considered and focus on the case with only 2 receivers. We use a curve-fitting approach based on the simulation results to get a closed-form formula of the packet loss probability with 2 receivers. This curve-fitting approach is also applicable to other cases in which more receivers are leveraged by MRC. The formula is easy to use and accurate when $\gamma \in[3.3,4.5]$ and the loss probability is greater than $0.5 \%$. For pure Aloha when advanced transmission techniques (e.g., interleaving, robust channel coding, etc.) are applied and when the capture ratio is $3 \mathrm{~dB}$, the system capacity for a $10 \%$ packet loss probability is increased by a factor greater than 1.26 compared to a simple scheme in which each base station independently decodes packets.
\end{abstract}

\section{Introduction}

Low Power Wide Area Network (LPWAN) is regarded as a promising solution to handle future machine type communication (MTC) traffic. There are several technologies, but most representative ones (Sigfox and LoRaWAN) are based on Aloha because of its simplicity [1].

The radio access network (RAN) of LPWAN supports macro reception diversity : a packet transmitted by a given device can be received by several base stations (BS). In the standard diversity scheme, each BS autonomously and independently decodes the packets and then sends the decoded packets to the core network. A packet is successfully delivered if at least one BS decodes the packets. This scheme is referred to as selection-combining-based macro diversity, simply written as SC macro diversity. It is currently used by Sigfox and LoRaWAN [1]. In [2] we analyzed the performance of Aloha and showed that the capacity of pure Aloha with macro

${ }^{*}$ published in Telecommunication Systems

${ }^{\dagger}$ Authors are from IMT Atlantique/IRISA, 2 rue de la Chataigneraie, 35510 Cesson Sevigne, France 
diversity is at least 2 times as much as that in systems in which a device transmits only to the best base station when the path loss exponent is 4 , the shadowing standard deviation is $8 \mathrm{~dB}$ and the target packet loss rate is $10 \%$

\subsection{Maximum ratio combining (MRC) in LPWAN}

We wonder whether this capacity can be further increased with more advanced combining techniques. We thus propose to use another network architecture that implements macro reception diversity, as illustrated in Fig. 1. Its principle is similar to the centralized RAN of cellular networks : a radio unit (RU) is installed at each site. The RU converts the received radio signal into a baseband signal, digitizes it and transmits it to the central unit via a fronthaul link. The central unit is responsible for the demodulation and decoding process. Thus, it is possible to linearly combine the signals from different RUs and to use maximum ratio combining (MRC) techniques to decode the packet. A series of MRC function blocks are installed within the central unit so that several transmitted packets in the radio network can be simultaneously decoded. This scheme is referred to as MRC macro diversity. With such an architecture, there are three

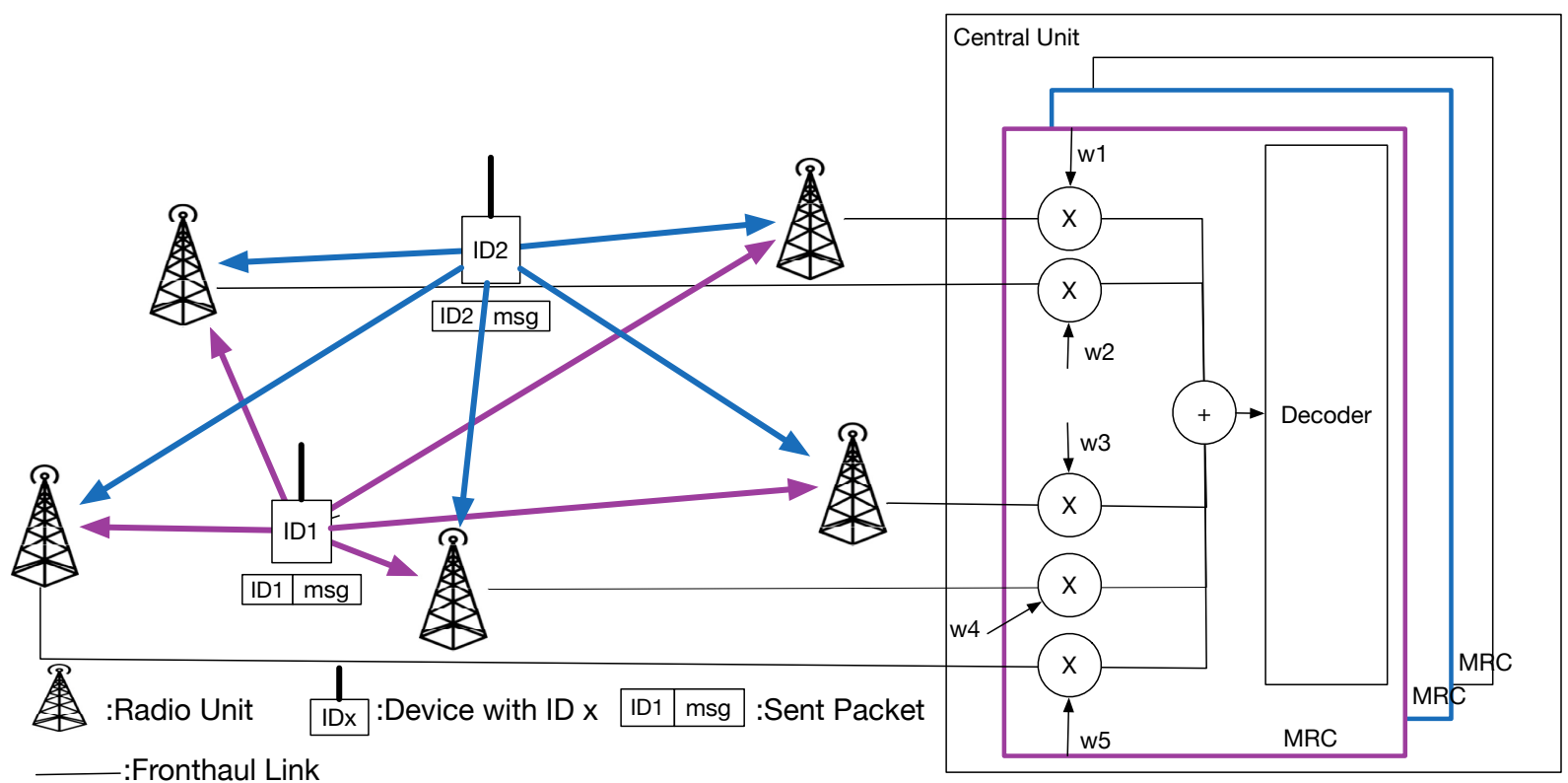

FIgURE 1 - LPWAN with MRC macro diversity

main issues : synchronization of the upstream, determination of the weight of each branch in the MRC, and load on the fronthaul. In LPWAN, each packet starts with a sequence that has good auto-correlation properties. This makes synchronization of the upstream and estimation of the Signal-to-Interference-and-Noise Ratio (SINR) on each branch possible. In some cases, the bitrate is so low that the symbol time (10 ms in Sigfox) is larger than the propagation delay and no re-synchronisation is necessary. Due to the low bitrate provided by LPWAN, the capacity required on the fronthaul is moderate : for Sigfox, there are 192 channels of $100 \mathrm{~Hz}$, which gives $192 \times 100 \times 2 \times 16 \times 2 \approx 1.2 \mathrm{Mbit} / \mathrm{s}$ for the fronthaul with a 2 over-sampling factor and 16-bit quantization of both the in-phase and quadrature components. It is thus not fundamentally impossible to use MRC macrodiversity in LPWAN, though the cost of development and deployment is not negligible. 


\subsection{Related works and motivations}

Due to its simplicity, Aloha has regained the focus of research community in recent years for wide range of application scenarios. For example, Sarker et al. [3] analyzed the performance of half-duplex slotted Aloha protocol in terms of throughput, packet rejection probability and selfstability, in infrastructure-less networks with/without capture and retransmission cut-off scheme. Ghanbarinejad et al. [4] studied distributed probabilistic access within networks supporting multipacket reception (MPR) at the physical layer. A throughput-optimal medium access strategy was proposed and analyzed. The analysis about pure Aloha is not covered in $[3,4]$. It is worth noting that pure Aloha protocol is attractive for LPWAN, because synchronizing all terminals would require extra payload and energy-consuming transmissions and thus increases the manufacture cost of terminals. The authors of [5] confirmed the interest of applying pure Aloha with receiver diversity in wide range sensor networks and proposed an analytic model for packet interference calculation in asynchronous random access protocols using diversity. The proposed model allows to evaluating the packet loss rate and throughput without time-consuming simulations. Their proposed model also servers as the ground base for further studies in which iterative interference cancellation is applied to received frames. However, the spatial distribution of transmitters and receivers have not been considered.

Combining techniques can be leveraged to improve the performance of the basic standard Aloha protocol. The research efforts about applying combining techniques to improve the performance of cellular networks can be backed to the years of 1990s. For example, the performance of SC macro diversity and MRC macro diversity was studied for GSM networks with a remote antenna system by using hexagonal topology [6], but analytical formulas were not available. A comprehensive framework for performance of cooperative wireless systems based on MRC has recently been proposed in [7], but the interference from other transmitters has not been considered. Similarly, the impact of spatial distribution of devices has not been considered. To tackle with this issue, stochastic geometry [8] was introduced into the research of wireless communication. It has served as a powerful tool to study the performance of ad-hoc [9] and cellular networks [10] but can also be used for LPWAN. In this field, SC diversity loss due to interference correlation in Poisson networks with multi-antenna receivers was studied by [11]. The performance of MRC in the presence of spatially correlated interference across antennas was characterized in [12]. However, MRC studied in [12] was for multi-antenna arrays deployed on the same BS, which is not the same case as the one we consider.

To the best of our knowledge, no existing work in the literature gives a performance analysis of MRC macro diversity when it is applied to random access.

\subsection{Contributions and organization}

In this paper we evaluate the performance of Aloha with MRC macro diversity in terms of packet loss probability in relation to the system load. We find a closed-form analytical model that is easy to use and takes into account the most important propagation parameters. Such a model is obtained by combining stochastic based analysis and curve fitting techniques. With the analytical model, an operator can deduce the capacity of a system without and with MRC, which is defined as the maximum load that provides a given loss rate target. Determining the gain of MRC is important to check if the investment related to this technology is justified.

The rest of this paper is organized as follows : Sec. 2 introduces the system model and some existing research efforts in stochastic geometry that serve as the basis of analysis in the following 
section. Sec. 3 details the analysis about the packet loss rate of MRC macro diversity with the interference and SIR independence assumption. Sec. 4 study the impact of the aforementioned assumption and discuss the performance of MRC under different receiver settings by simulations. Sec. 5 proposes a closed-form analytical model for 2 receivers MRC in which the parameters can be obtained by curve fitting approach. The model is based on the insights obtained from analysis in Sec. 3. In Sec. 6, we discuss the correctness of our curve fitting based analytical model proposed in Sec. 5 with respect to simulation results and study the system capacity gain of MRC compared with SC. Sec. 7 concludes this paper.

\section{System model}

For any random variable $(\mathrm{RV}) X$, let $f_{X}(x), F_{X}(x), \mathcal{L}_{X}[s], \phi_{X}(\omega)$ be its probability density function $(\mathrm{PDF})$, cumulative distribution function (CDF), Laplace transform (LT) and characteristic function $(\mathrm{CF})$, respectively.

\subsection{Distribution of Nodes and Aloha protocols}

The system is made of a two-dimension infinite wireless network with only uplink transmissions. The access protocol is either pure Aloha (P-Aloha) or slotted Aloha (S-Aloha). All devices randomly transmit packets of fixed duration $T_{\text {slot }}$ with probability $p$ and may generate interference to each other. Following the approach of [13], we consider a time-space point process $\Psi=\left\{\left(X_{i}, T_{k}\right)\right\}$ where $X_{i} \in \mathbb{R}$ denotes the location of the device that sends a packet during time interval $\left[T_{k}, T_{k}+T_{\text {slot }}\right)$. We assume that $\Psi$ form a homogeneous Poisson point process (PPP) both in time and space with intensity $p \lambda_{m}$. Similarly, the locations of RUs form a homogeneous PPPs denoted by $\Phi$ with intensity $\lambda_{b}$. We define the normalized load (per RU) as $L=p \lambda_{m} / \lambda_{b}$.

\subsection{Propagation model}

The propagation model is based on Okumura-Hata with both shadow and Rayleigh fading. We assume that MTC devices transmit with unit power level. The received power $P_{r}$ of a packet at the RU is given by :

$$
P_{r}=r_{g}{ }^{-\gamma} H 10^{\sigma_{\mathrm{dB}}} \chi / 10=r_{g}{ }^{-\gamma} H \exp \left(\sigma_{\mathrm{dB}} \frac{\ln (10)}{10} \chi\right),
$$

where $r_{g}$ refers to the Euclidean distance between the transmitter and the receiver, $\gamma$ is the path-loss exponent, $H$ is an exponentially distributed RV with mean 1, which models Rayleigh fading, and $\chi$ is a standard normal variable. This means that term $10^{\sigma_{\mathrm{dB}}} \chi / 10$ represents $\log$ normal shadowing effect. For the sake of simplification, we define $\sigma=\sigma_{\mathrm{dB}} \ln (10) / 10$. We assume that $H$ and $\chi$ are both constant during a packet transmission, and mutually independent for different links.

\subsection{Displacement Theorem}

To facilitate the analysis, we use the displacement theorem, which is formulated as a lemma in $[14$, lemma 1]. 
Lemma 1. For a homogeneous PPP $\Phi \subset \mathbb{R}^{2}$ with spatial density $\lambda_{b}$, if each point $r_{g} \in \Phi$ is transformed to $r \in \mathbb{R}^{2}$ such that $r=X^{-\frac{1}{\gamma}} r_{g}$, where $\{X\}$ are i.i.d., such that $\mathbb{E}\left[X^{\frac{2}{\gamma}}\right]<+\infty$, the new point process $\Phi^{\prime} \subset \mathbb{R}^{2}$ defined by the transformed points $r$ is also a homogeneous PPP with density $\lambda_{b}^{\prime}=\lambda_{b} \mathbb{E}\left[X^{\frac{2}{\gamma}}\right]$.

We thus rewrite (1) as follows :

$$
P_{r}=H r^{-\gamma}, \text { with } r=\exp (-\chi / \gamma) r_{g},
$$

which indicates that the performance analysis in the initial PPP with shadowing is equivalent to that in a transformed PPP of intensity $\lambda_{b}^{\prime}=\lambda_{b} \mathbb{E}\left[e^{\frac{2}{\gamma} \chi}\right]=\lambda_{b} e^{\frac{2 \sigma^{2}}{\gamma^{2}}}$ without shadowing but with the modified distance $r$. In the following, all distances are the modified ones in the transformed PPP.

\subsection{Packet loss model}

The analysis is made on a system level. Hence, as considered by a lot of authors, we do not model the detail of the digital transmission chain. A bit is assumed to be correctly received if the Signal to Noise and Interference ratio (SINR) is not less than a threshold value. Our objective is to find the maximum capacity of the system and we thus consider it in high load conditions : interferences are much larger than the background noise. Thus, the transmission success probability $p_{b s}$ for a transmit-receiver pair with distance $r$ is defined as follows :

$$
p_{b s}(r)=\mathbb{P}\left\{\frac{P_{r}}{I} \geq \theta_{T}\right\},
$$

where $I$ refers to the cumulative interference and $\theta_{T}$ is the minimum SINR to ensure propre reception.

Using (2) we can re-write (3) as

$$
p_{b s}(r)=\mathbb{P}\left\{H \geq I r^{\gamma} \theta_{T}\right\}=\mathcal{L}_{I}\left(r^{\gamma} \theta_{T}\right) .
$$

In (4), each BS has its own receiving and decoding chain. In section 3, we consider a global receiver at the system level as shown in figure 1, which modifies the expression of the success probabilities but still requires the computation of the cumulative interference. In the following, we analyse this interference.

\subsection{Interference analysis}

Consider a device $x_{0}$ that is transmitting at time $t$. Without loss of generality, we can assume the device is located at the origin. The distance to $\mathrm{RU} y_{j}$ is $r_{y_{j}}$. From the propagation model and displacement theorem, the cumulative interference at RU $y_{j}$ can be written as follows :

$$
I_{y_{j}}(t)=\sum_{x_{i} \in \Psi(t) \backslash\left\{x_{0}\right\}} H_{x_{i}, y_{j}} r_{x_{i}, y_{j}}^{-\gamma},
$$

where $H_{x_{i}, y_{j}}$ is the Rayleigh fading for the link between $x_{i}$ and $\mathrm{RU} y_{j}, r_{x_{i}, y_{j}}$ refers to distance from the interfering device $x_{i}$ to BS $y_{j}, \Psi(t)$ is the spatial PPP formed by devices transmitting at time $t$. 


\subsubsection{Slotted Aloha}

In slotted Aloha, all transmitters are synchronised on a common slot structure. The propagation delay is assumed to be very small compared to the slot duration $T_{\text {slot }}$. The cumulative interference is thus constant within each slot $\left(I_{y_{i}}(t)=I_{y_{i}}\left(T_{k}\right)\right.$ if $t \in\left[T_{k}, T_{k}+T_{\text {slot }}\right)$ for any slot $k$ ) and is i.i.d over successive slots. The Laplace transform of cumulative interference with independent Rayleigh fading and log-normal shadowing was calculated in [15]. We denote it as $I$ for the sake of simplicity :

$$
\mathcal{L}_{I}(s)=\exp \left\{-p \lambda_{m} \pi A \exp \left(2 \sigma^{2} / \gamma^{2}\right) s^{2 / \gamma}\right\}
$$

where $A=\Gamma\left(1-\frac{2}{\gamma}\right) \Gamma\left(1+\frac{2}{\gamma}\right)$ and $\Gamma(z)=\int_{0}^{+\infty} x^{z-1} e^{-x} d x$.

\subsubsection{Pure Aloha with average interference}

In pure Aloha, there is no synchronization. Let $T$ be the instant of time at which device $x_{0}$ starts the packet transmission. The cumulative interference $I_{y_{i}}(t)$ in interval $\left[T, T+T_{\text {slot }}\right]$ is variable during the packet transmission. When advanced transmission techniques (e.g., interleaving, robust channel coding, etc.) are used, the probability to decode a packet is a function of the average interference $I_{y_{i}}^{\text {mean }}=\frac{1}{T_{\text {slot }}} \int_{T}^{T+T_{\text {slot }}} I_{y_{i}}(t) d t$. This configuration is called $P a$-Aloha (pure Aloha with average interference). LoRa uses forward error correction (FEC) with different coding rates and can be considered as a Pa-Aloha system. Our model applies for one coding rate. The value of the capture ratio threshold $\theta_{T}$ is determined according to the chosen coding rate.

As shown in $\left[13\right.$, Sec.2.4], formula (6) can be reused for $I_{y_{i}}^{\text {mean }}$ by letting $A=\frac{2 \gamma}{\gamma+2} \Gamma(1-$ $\left.\frac{2}{\gamma}\right) \Gamma\left(1+\frac{2}{\gamma}\right)$.

\subsubsection{Pure Aloha with maximum interference}

In low-cost systems, there is neither error correction nor interleaving techniques : the packet is delivered if and only if each bit is correctly received. We denote this case as PmAloha (pure Aloha with maximum interference). For example, Sigfox only includes an error detection code and can be regarded to use Pm-Aloha. The SINR should be larger than or equal to $\theta_{T}$ during $T_{\text {slot }}$. The packet success probability is a function of maximum interference $I_{y_{i}}^{\max }=\max _{t \in\left[T, T+T_{\text {slot }}\right]} I_{y_{i}}(t)$. According to [13, Sec.2.4], there is no closed-form for $I^{\max }$, and the authors use a simulation approach to study pm-Aloha. In [2, Sec.III-B], we propose to approximate $I_{y_{j}}$ by an upper bound, which is the sum of interference levels at the start and end time of the considered packet transmission. With our proposed upper bound, (6) is extended to cover the case of Pm-Aloha with $A=2 \Gamma(1-2 / \gamma) \Gamma(1+2 / \gamma)$. 


\subsubsection{Unified expression of the cumulative interference for all cases}

We can use (6) to calculate $\mathcal{L}_{I}(s), \mathcal{L}_{I^{\text {mean }}}(s), \mathcal{L}_{I^{\max }}(s)$ for S-Aloha, Pa-Aloha and Pm-Aloha, respectively, with

$$
A= \begin{cases}\Gamma\left(1-\frac{2}{\gamma}\right) \Gamma\left(1+\frac{2}{\gamma}\right), & \text { for S-Aloha } \\ \frac{2 \gamma}{\gamma+2} \Gamma\left(1-\frac{2}{\gamma}\right) \Gamma\left(1+\frac{2}{\gamma}\right), & \text { for Pa-Aloha } \\ 2 \Gamma\left(1-\frac{2}{\gamma}\right) \Gamma\left(1+\frac{2}{\gamma}\right), & \text { for Pm-Aloha }\end{cases}
$$

\section{Packet loss probability with the interference and SIR inde- pendence assumption}

\subsection{General formula of the packet loss probability}

Let $\theta_{y_{j}}$ be the received signal-to-interference ratio (SIR) at $y_{j}$ and $\epsilon_{y_{j}}=H_{y_{j}} / I_{y_{j}}$. The SIR $\theta_{y_{j}}$ at $\mathrm{RU} y_{j}$ is :

$$
\theta_{y_{j}}=H_{y_{j}} r_{y_{j}}^{-\gamma} / I_{y_{j}}=\epsilon_{y_{j}} r_{y_{j}}^{-\gamma} .
$$

As proved in section 14.4.1 of [16], the received SIR $\Theta$ with MRC is :

$$
\Theta=\sum_{y_{j} \in \Phi_{b}} \theta_{y_{j}}=\sum_{y_{j} \in \Phi_{b}} \epsilon_{y_{j}} r_{y_{j}}^{-\gamma}
$$

Let $P_{f, \mathrm{mrc}}$ be the packet loss probability with MRC, which is our main quality indicator. Retransmission is not considered in our work. Thus, a packet is lost if and only if $\Theta$ is less than a threshold $\theta_{T}$. Hence,

$$
P_{f, \mathrm{mrc}}=\mathbb{P}\left\{\Theta<\theta_{T}\right\}=F_{\Theta}\left(\theta_{T}\right) .
$$

This probability is numerically derived from its corresponding $\mathrm{CF} \phi_{\Theta}(\omega)$, which itself can be obtained from $\mathcal{L}_{\Theta}(s)$.

\subsection{Characteristic function of the packet loss probability}

By definition, $\mathcal{L}_{\Theta}(s)=\mathbb{E}\left[e^{-s \Theta}\right]$ and using (9), we get :

$$
\mathcal{L}_{\Theta}(s)=\mathbb{E}\left[\exp \left(-s \sum_{y_{j} \in \Phi_{b}} \epsilon_{y_{j}} r_{y_{j}}^{-\gamma}\right)\right]
$$

RV $\epsilon_{y_{j}}=H_{y_{j}} / I_{y_{j}}$ are identically distributed but mutually dependent, because the cumulative interference at two different RU are mutually dependent : when a device is transmitting, it generates some non-negligible interference on RU that are not very far. However, it is still rational to assume that the interference received by different $R U$ are mutually independent, because the interference correlation coefficient is shown to be close to 0 if locations of two RU are different with path loss model $r^{-\gamma}$ [15, Sec.3.8.1, lemma 3.5]. It is worth indicating that 
packet loss rate obtained under independent interference hypothesis is lower than that obtained with correlated interferences. More discussion is given in Sec. 4. We thus have :

$$
\mathcal{L}_{\Theta}(s)=\mathbb{E}\left[\prod_{y_{j} \in \Phi_{b}} \mathbb{E}_{\epsilon_{y_{j}}}\left[\exp \left(-s \epsilon_{y_{j}} r_{y_{j}}^{-\gamma}\right)\right]\right] .
$$

Applying Probability Generating FunctionaL (PGFL) of PPP $\Phi_{b}$ to (12), which states for some function $f(x)$ that $\mathbb{E}\left[\prod_{x \in \Phi} f(x)\right]=\exp \left(-\lambda\left(\int_{\mathbb{R}^{2}}(1-f(x)) d x\right)\right)$, and changing the order of integration and expectation operator, we have :

$$
\begin{aligned}
\mathcal{L}_{\Theta}(s) & =\exp \left\{-\int_{0}^{+\infty} \mathbb{E}_{\epsilon}\left[1-\exp \left(-s \epsilon r^{-\gamma}\right)\right] 2 \pi \lambda_{b}^{\prime} r d r\right\} \\
& =\exp \left\{-\mathbb{E}_{\epsilon}[\underbrace{\int_{0}^{+\infty}\left(1-\exp \left(-s \epsilon r^{-\gamma}\right)\right) 2 \pi \lambda_{b}^{\prime} r d r}_{D}]\right\} .
\end{aligned}
$$

Let us focus on integral $D$ :

$$
\begin{aligned}
D & \stackrel{(\mathrm{a})}{=} \pi \lambda_{b}^{\prime} \int_{0}^{+\infty}(1-\exp (-x)) d\left(-x^{-\frac{2}{\gamma}}(s \epsilon)^{\frac{2}{\gamma}}\right) \\
& \stackrel{(\mathrm{b})}{=} \pi \lambda_{b}^{\prime} \int_{0}^{+\infty} \exp (-x) x^{-\frac{2}{\gamma}}(s \epsilon)^{\frac{2}{\gamma}} d x \\
& =\pi \lambda_{b}^{\prime}(s \epsilon)^{\frac{2}{\gamma}} \Gamma\left(1-\frac{2}{\gamma}\right),
\end{aligned}
$$

where step $(a)$ is obtained via a change of variable $x=s \epsilon r^{-\gamma}$, step $(b)$ is achieved via integration by parts.

Combining (13) and (14), $\mathcal{L}_{\Theta}(s)$ is finally simplified as :

$$
\begin{gathered}
\mathcal{L}_{\Theta}(s)=\exp \left(-\lambda_{b}^{\prime} \pi \mathbb{E}\left[\epsilon^{\frac{2}{\gamma}}\right] \Gamma\left(1-\frac{2}{\gamma}\right) s^{\frac{2}{\gamma}}\right) \\
\stackrel{(a)}{=} \exp \left(-\lambda_{b}^{\prime} C \mathbb{E}\left[I^{-\frac{2}{\gamma}}\right] s^{\frac{2}{\gamma}}\right),
\end{gathered}
$$

where step (a) is obtained by $\mathbb{E}\left[\epsilon^{\frac{2}{\gamma}}\right]=\Gamma\left(1+\frac{2}{\gamma}\right) \mathbb{E}\left[I^{-\frac{2}{\gamma}}\right]$ and constant $C=\pi \Gamma(1-\gamma / 2) \Gamma(1+\gamma / 2)$.

Computing $\mathbb{E}\left[I^{-\frac{2}{\gamma}}\right]$ in (15) is a fractional moment calculation problem. It is known that $n^{\text {th }}$-order (i.e. any positive integer order) moment of random variable $X$ can be obtained from its LT :

$$
\mathbb{E}\left[X^{n}\right]=\left.(-1)^{-n} \mathcal{L}_{X}^{(n)}(s)\right|_{s=0},
$$

if and only if $\left.\mathcal{L}_{X}^{(n)}(s)\right|_{s=0}$ exists. This conclusion has been extended to real number domain and is expressed as follows [17] :

$$
\mathbb{E}\left[X^{a}\right]=\left.(-1)^{-a}{ }_{s} D_{\infty}^{a}\left[\mathcal{L}_{X}(s)\right]\right|_{s=0},
$$

where $a$ is a real number and ${ }_{s} D_{\infty}^{a}\left[\mathcal{L}_{X}(s)\right]$ refers to a fractional derivative if $a$ is positive, otherwise a fractional integral. Any fractional moment of LT can be obtained with (17) if and only if ${ }_{s} D_{\infty}^{a}\left[\mathcal{L}_{X}(s)\right]$ exists when $s=0$. 
To calculate $\mathbb{E}\left[I^{-\frac{2}{\gamma}}\right]$, the fractional integral on $\mathcal{L}_{X}[s]$ should be considered. Since LT is only defined on the positive axis, its fractional integral is dfined as follows [17] :

$$
{ }_{s} D_{\infty}^{a}\left[\mathcal{L}_{X}(s)\right]=\frac{(-1)^{a}}{\Gamma(-a)} \int_{s}^{+\infty}(u-s)^{-a-1} \mathcal{L}_{X}(u) d u,
$$

From (17) and (18), we have :

$$
\mathbb{E}\left[X^{a}\right]=\left.(-1)^{-a}{ }_{s} D_{\infty}^{a}\left[\mathcal{L}_{X}(s)\right]\right|_{s=0}=\frac{1}{\Gamma(-a)} \int_{0}^{+\infty} u^{-a-1} \mathcal{L}_{X}(u) d u
$$

Substituting $X=I, a=-2 / \gamma$ and (6) into (19), we have :

$$
\mathbb{E}\left[I^{-\frac{2}{\gamma}}\right]=\frac{1}{\Gamma(2 / \gamma)} \int_{0}^{+\infty} u^{2 / \gamma-1} \exp \left\{-p \lambda_{m} \pi A \exp \left(2 \sigma^{2} / \gamma^{2}\right) u^{2 / \gamma}\right\} d u
$$

After some elementary steps, we get :

$$
\mathbb{E}\left[I^{-\frac{2}{\gamma}}\right]=\left(\Gamma(1+2 / \gamma) p \lambda_{m} \pi A \exp \left(2 \sigma^{2} / \gamma^{2}\right)\right)^{-1}
$$

With (15), (21) and the expression of $\lambda_{b}^{\prime}$, the closed-form expression of $\mathcal{L}_{\Theta}(s)$ is :

$$
\mathcal{L}_{\Theta}(s)=\exp \left(-\Gamma(1-2 / \gamma)(A L)^{-1} s^{\frac{2}{\gamma}}\right),
$$

where $L=p \lambda_{m} / \lambda_{b}$ is the normalized load per RU.

Using $\phi_{\Theta}(\omega)=\mathcal{L}_{\Theta}(s)$ with $s=-i \omega\left(\right.$ where $\left.i^{2}=-1\right)$, we get :

$$
\phi_{\Theta}(\omega)=\exp \left(-\Gamma(1-2 / \gamma)(A L)^{-1} e^{-i \pi / \gamma} \omega^{\frac{2}{\gamma}}\right)
$$

We observe that the packet loss probability of MRC depends on normalized load, path loss exponent and SIR threshold, but has nothing to do with shadowing effect.

A numerical technique is presented in $[18,19]$ to calculate $F_{\Theta}(\theta)$ from $\phi_{\Theta}(\omega)$. Its key idea is to calculate the Fourier transform of $e^{-\eta \theta} F_{\Theta}(x)$ where $e^{-\eta \theta}$ is a damping function with $\eta>0$.

$$
\int_{-\infty}^{+\infty} e^{i \omega \theta} e^{-\eta \theta} F_{\Theta}(\theta) d x=\frac{1}{\eta-i \omega} \phi_{\Theta}(\omega+i \eta)
$$

Applying Fourier inversion for (24), we get :

$$
F_{\Theta}(\theta)=\frac{e^{\eta \theta}}{\pi} \Re\left\{\int_{0}^{+\infty} \frac{e^{-i \omega \theta}}{\eta-i \omega} \phi_{\Theta}(\omega+i \eta) d \omega\right\},
$$

which allows us to numerically calculate $P_{f, \text { mrc }}$ by combining (10) and (23).

\subsection{Packet loss probability for $\gamma=4$}

The combination of (10), (23) and (25) establishes a numerical framework allowing numerically calculating the loss probability. In this section, we concentrate on a special case where $\gamma=4$, because this is a case (and to the best of our knowledge, the only case) in which the closed-form expression of loss probability is available. 
It is known that an RV $X$ following Levy distribution can be characterized by two parameters : location parameter $U$ and scale parameter $V$. If $U=0$, its $\operatorname{CDF} F_{X}(x)$ and LT $\mathcal{L}_{X}(s)$ are respectively :

$$
\begin{aligned}
& F_{X}(x)=1-\operatorname{erf}\left(\sqrt{\frac{V}{2 x}}\right), \\
& \mathcal{L}_{X}(s)=\exp (-\sqrt{2 V s}),
\end{aligned}
$$

When $\gamma=4,(22)$ can be written as :

$$
\mathcal{L}_{\Theta}(s)=\exp \left(-\sqrt{\pi}(A L)^{-1} s^{\frac{1}{2}}\right) .
$$

By comparing (27) and (28), we observe that RV $\Theta$ follows a Levy distribution with $U=0$ and $V=\pi /\left(2 A^{2} L^{2}\right)$. We obtain the closed-form expression of $P_{f, m r c}$ :

$$
P_{f, m r c}=1-\operatorname{erf}\left(\frac{\sqrt{\pi}}{2 A \sqrt{\theta_{T}} L}\right)
$$

where $A$ is given in (7). Note that for Pm-Aloha, (29) gives a close upper bound of the loss probability.

\section{Preliminary study based on simulations}

\subsection{Study of SIR independence hypothesis}

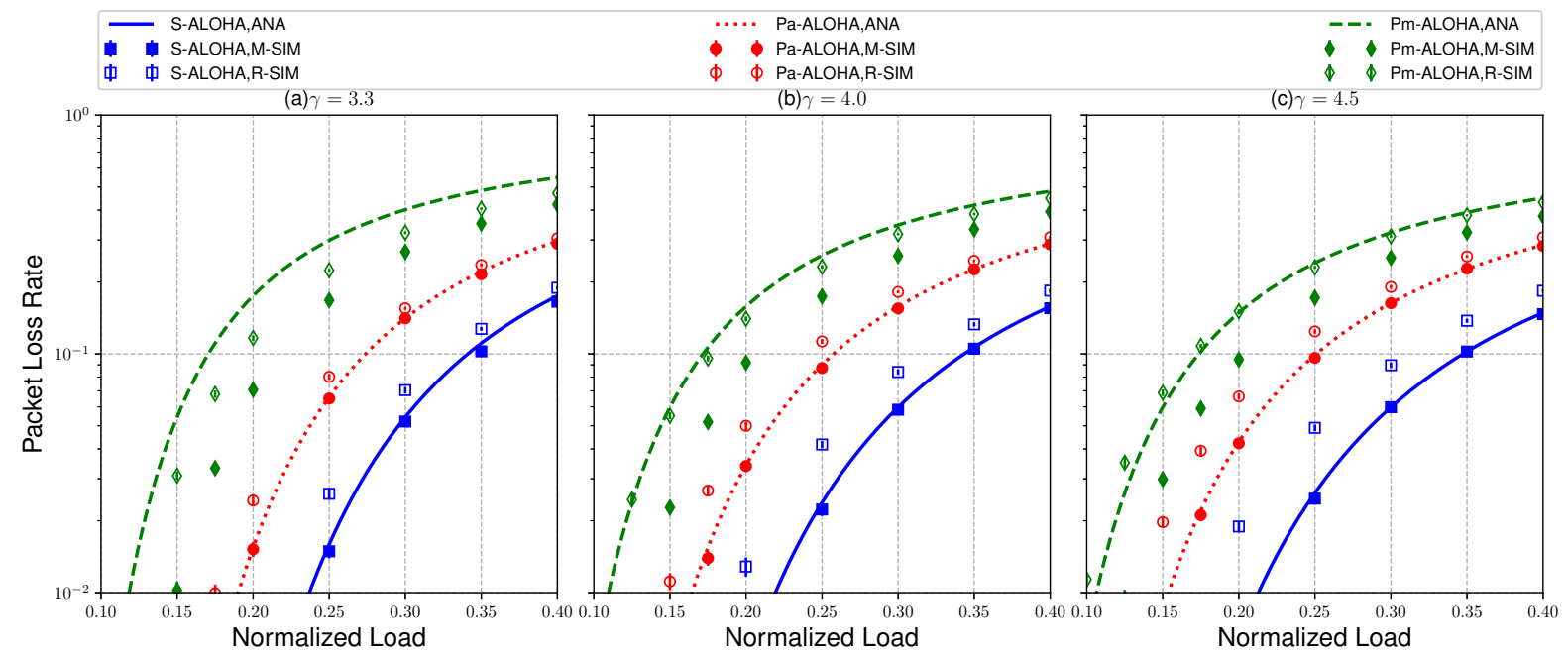

FiguRE 2 - Network packet loss rate with respect to $L$ for $\gamma=3.3,4.0,4.5$ (from left to right)

In this section, we study the impact of SIR independence hypothesis by simulation. We simulated different types of Aloha on an area of $100 \times 100 \mathrm{~km}^{2}$ and 500 RUs. Such a huge simulation area help eliminate the boundary effect due to a finite area. With 500 RUs on average in the considered area ensures that macro diversity is visible. The shadowing effect is $\sigma_{\mathrm{dB}}=8$ $\mathrm{dB}$ and capture ratio is $\theta_{T}=3 \mathrm{~dB}$. The number of devices is selected such that the normalized load $L$ varies from 0.1 to 0.5 . We first considered a realistic simulation, denoted by R-SIM, in 
which the interference on each RU is computed without any independence assumption. We also developed a model-based simulation, denoted by M-SIM. In M-SIM, we consider independent interference for different RUs, which is compliant with our numerical framework.

Fig. 2 gives the numerical results (i.e. ANA in the legend) and the simulation results for $\gamma=3.3,4.0,4.5$ and for packet loss rates in $[0.01,0.2]$, which is the interval of interest. The $95 \%$ confidence intervals are shown for each simulation but are so small that they are not visible. M-SIM results fit well with those given by the numerical framework for S-Aloha and Pa-Aloha. The formula proposed for Pm-Aloha (i.e. (7)) is an upper bound. This explains that the loss given by the analysis is always larger than the one given by M-SIM.

When $\gamma=3.3$ (refer to Fig. 2(a)), we observe a gap between R-SIM and M-SIM that is progressively reduced when the normalized load $L$ increases. When $L$ is greater that 0.3 , the disparity between two types of simulations, for Pa-Aloha, is negligible. The reason is that heavy load and the randomness due to the lack of synchronization both make the SIR independence hypothesis reasonable. For S-Aloha, the gap between R-SIM and M-SIM is negligible when $L>0.4$. This is because with S-Aloha, the SIR hypothesis is rational only in a high regime of load.

Comparing the three subfigures in Fig. 2, we observe that at the same load level, the difference between R-SIM and M-SIM are larger when the path loss exponent $\gamma$ increases. Thus, path loss exponent also has impact for interference-independence assumption. The larger the path loss exponent, the more important the effect of the interference correlation. This effect is also observed in [11].

For Pm-Aloha, in all cases, the simulation results are higher than that from numerical framework, because the analysis (i.e. (7)) is based on the upper bound approximation. Such an approximation is proposed by [2] to analyze SC macro diversity. The summation of SIR in MRC (i.e. Eq. (9)) makes the cumulated error brought by upper bound higher than that of SC macro diversity (refer to Fig.2 in [2]). However, we observe that the gap between analytical framework and R-SIM is negligible when $\gamma \in[4.0,4.5]$. The reason is that for such an interval, the interference correlation leads to the increase of the packet loss rate. This degradation compensates the error due to the use of an upper bound.

\subsection{Study of Involved RUs in MRC}

Recall that in the analysis detailed in Sec. 3, MRC mechanism benefits from the involvement of all RUs deployed on the considered infinite plane. This is actually impossible in real life. We would like to study the network packet loss probability of LPWAN network supporting MRC under different number of involved RUs. The simulation results are shown in Fig. 4.2. The capture ratio is $3 \mathrm{~dB}$, path loss exponent $\gamma=3.3$. We consider the following scenario according to the number of RUs that are leveraged by MRC : 1) only the best RU; 2) the two best RUs ; 3 ) the six best RUs ; 4) the twenty best RUs ; 5) all the RUs on the infinite plane. It is worth indicating that the first scenario with only the best RU involved into MRC mechanism is equivalent to the selection combination technique.

From Fig. 4.2, we observe that MRC has significant performance improvement if more than one RUs are leveraged by MRC. More participating RUs, more higher the performance gain. The case in which all the deployed RUs participate MRC procedure gives the upper bound. 


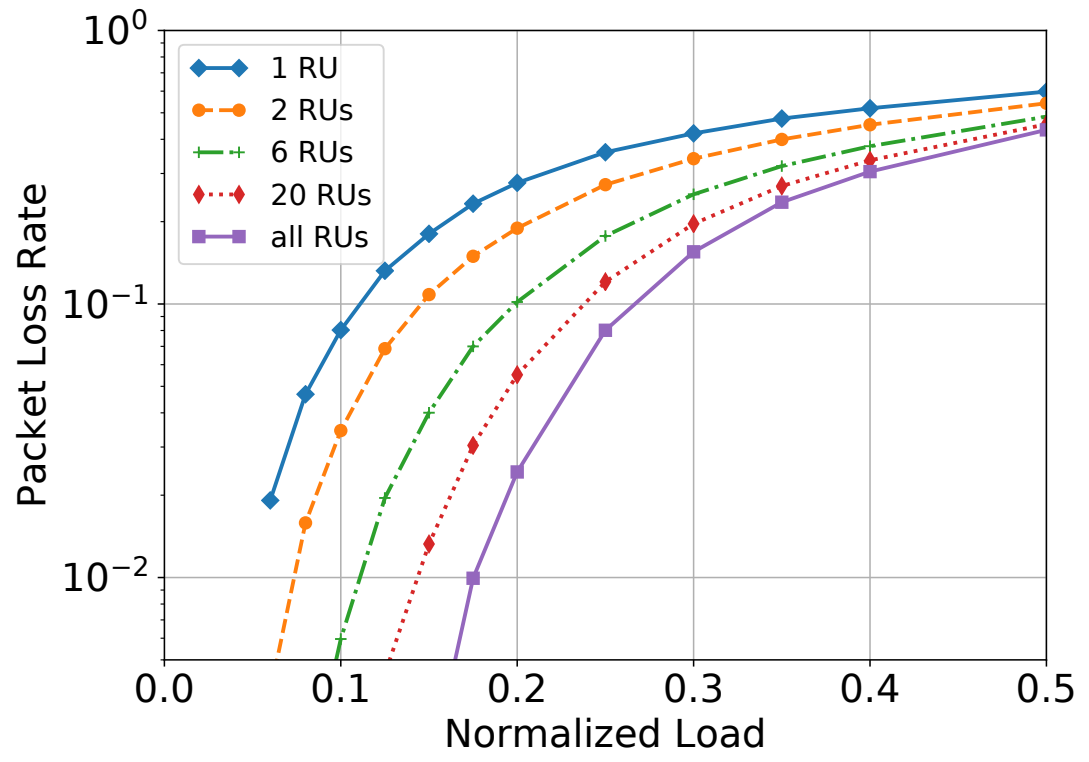

FiguRE 3 - Network packet loss rate under different radio units settings, $\gamma=3.3$

\section{Curve fitting with respect to simulation results}

\subsection{Presentation of the approach}

From previous section, what we can infer are as follows :

- The interference independence hypothesis has a limited applicable scope. It depends on the normalized load, path loss exponent and Aloha type. Usually, when the normalized load is at a moderate level, such a hypothesis does not hold and an obvious gap between R-SIM and M-SIM can be observed.

- To have an accuracy analysis, the intuitive solution is to analyze with dependent interference. However, it will be very difficult, even impossible, to obtain an analytical framework for packet loss rate.

- A closed-form expression for packet loss rate (e.g. equation (29)) when $\gamma=4.0$ and the evolution trends of M-SIM and R-SIM are similar although the gap between them is not neglected.

We thus assume that packet loss for $\gamma>2.0$ can be approximated by an expression similar to (29). The related parameters can be determined by curve fitting with respect to simulation results. This is a compromise solution that maintains the accuracy of analytical model and reduces the analysis complexity.

From the Laplace analysis (which is valid for any value of $\gamma>2$ ) and (22), we can deduce the following insights useful for curve fitting :

- the packet loss probability is a function of normalized load $L$ with path-loss exponent $\gamma$ and SINR threshold $\theta_{T}$ as parameters;

- the shadowing effect actually has no impact on the average packet loss rate;

- the impact of SINR threshold to packet loss probability is in form of $\theta^{\frac{2}{\gamma}}$. 
- the denominator of the input of error function is a linear function of $L$ (refer to (29)).

Thus, the packet loss rate for all values of $\gamma>2$ are assumed to have the following expression :

$$
P_{f, \mathrm{mrc}}(L)=1-\operatorname{erf}\left(\frac{1}{\left[K(\gamma) \theta_{T}^{\frac{2}{\gamma}} L+B(\gamma)\right]}\right),
$$

where $K(\gamma)$ and $B(\gamma)$ are parameters to be determined by curve fitting with respect to R-SIM simulation results.

A linear form about $L$ is obtained from (30):

$$
\left[\operatorname{erfc}^{-1}\left(P_{f, m r c}(L)\right)\right]^{-1}=K(\gamma) \theta_{T}^{\frac{2}{\gamma}} L+B(\gamma),
$$

where $\operatorname{erfc}^{-1}(\cdot)$ refers to the inverse function of the complementary error function.

\section{2 case study : $\mathrm{MRC}$ with 2 best RUs}

We take the case in which only the 2 RUs that receive the signal with the best SNRs are involved by MRC mechanism. The objective is to evaluate the gain provided by MRC in the simplest case. This study can also be seen as an example, to illustrate how parameter $K$ and $B$ are obtained by curve fitting. This methodology is applicable for other cases, with 3,6,20 RUs, or even all the RUs in the infinite plane (i.e. the ideal case).

From (31), for each $\gamma$, linear regression can applied to obtain the parameter $K$ and $B$. By varying $\gamma$ from [3.3,4.5], a series of $K, B$ are obtained for all type of Aloha. Fig. 5.2 shows the comparison of packet loss rate respectively obtained from realistic simulation (R-SIM) and curve-fitting-based model. The leftmost, central and rightmost columns respectively corresponds to S-Aloha, Pa-Aloha and Pm-Aloha. From this figure, we observe that for each $\gamma$, the packet loss can be well predicted by Eq. (30).

The metric to measure the accuracy of our proposed curve-fitting-based analytical model is mean absolute percentage error (MAPE), whose mathematical definition is as follows :

$$
\mathrm{MAPE}=\frac{100 \%}{N} \sum_{i=1}^{N}\left|\frac{\widehat{Y}_{i}-Y_{i}}{Y_{i}}\right|
$$

where $N$ refers to the number of different normalized loads used in simulation, $\widehat{Y}_{i}$ is packet loss rate obtained from fitted model corresponding to a certain level of load, $Y_{i}$ is the result from R-SIM simulation results. Different from other metrics such MSE (mean square error), MAPE is more suitable to our case, where the predicted value is in interval $[0,1]$.

Note that $K$ and $B$ they are also functions of $\gamma$. After plotting the evolution of $K, B$ with respect to $\gamma$, we observe that polynomial regression of 3 -order is sufficient to find the searched functions. The values of $K$ and $B$ under different $\gamma$ are obtained and shown in Tab. 1 . The value of MAPE is always smaller than $10 \%$, which confirms the validity of the approach. The fitted function for $K$ is as follows :

$$
K= \begin{cases}-0.0706 \gamma^{3}+1.067 \gamma^{2}-5.429 \gamma+10.95, & \text { for slotted Aloha } \\ -0.0613 \gamma^{3}+0.957 \gamma^{2}-4.945 \gamma+10.76, & \text { for pa-Aloha } \\ -0.0673 \gamma^{3}+1.076 \gamma^{2}-5.806 \gamma+13.475 & \text { for pm-Aloha }\end{cases}
$$



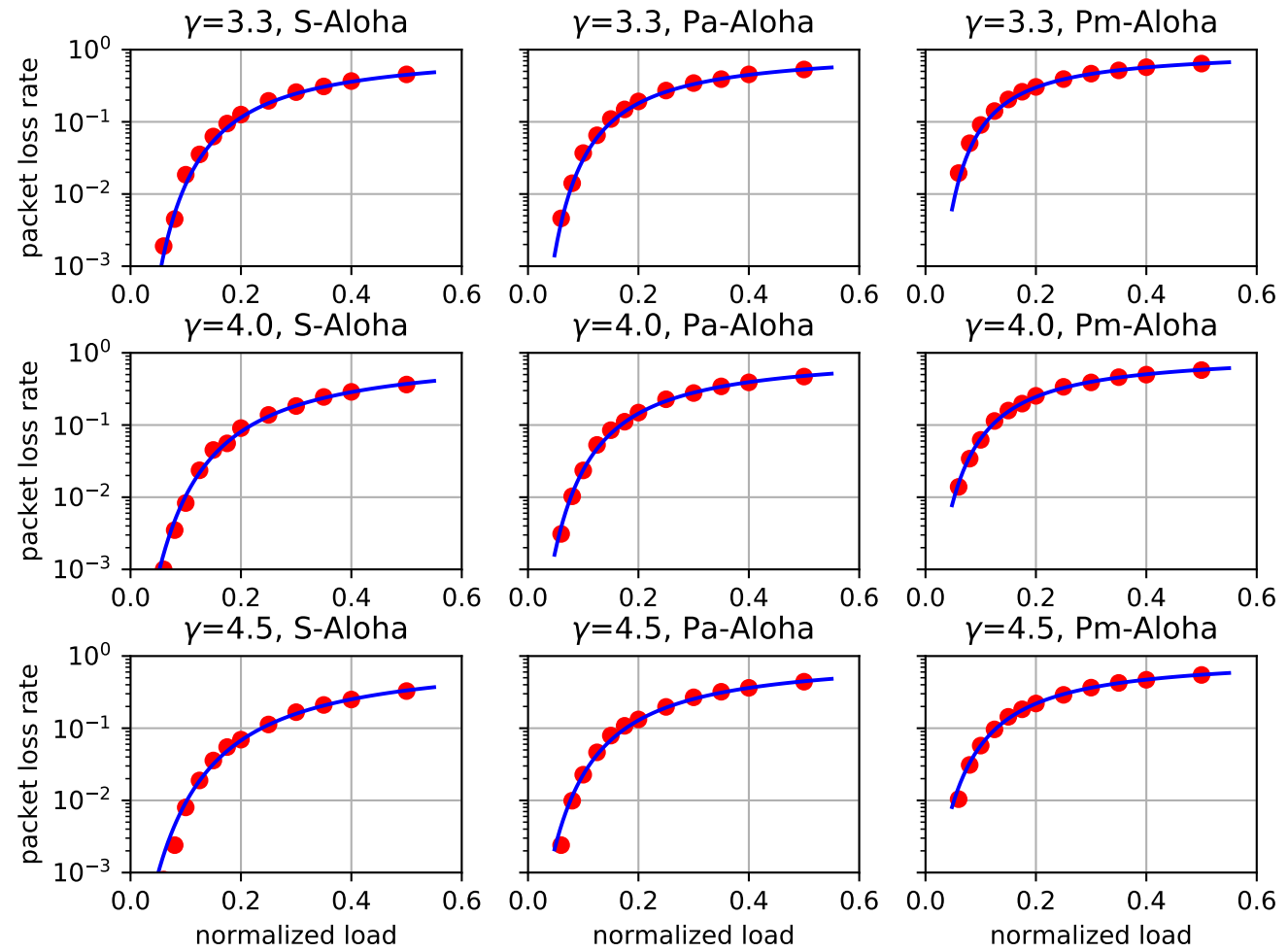

FiguRE 4 - Comparison between predicted value and realistic simulation results

TABLE 1 - Parameters obtained from curve fitting on the Pa-Aloha simulation results

\begin{tabular}{|c|c|c|c|}
\hline$\gamma$ & $\mathrm{K}$ & $\mathrm{B}$ & $\mathrm{MAPE}(\%)$ \\
\hline 3.3 & 2.651 & 0.2497 & 8.92 \\
\hline 3.5 & 2.5291 & 0.2578 & 8.97 \\
\hline 4.0 & 2.4242 & 0.2836 & 9.39 \\
\hline 4.5 & 2.2792 & 0.3109 & 5.52 \\
\hline
\end{tabular}


TABLE 2 - Error brought by the analytical formula $\left(L_{a}\right.$ and $L_{s}$ refer to the load for a given target loss from the formula and R-SIM simulation, respectively)

\begin{tabular}{|l|l|l|l|l|l|l|l|l|l|}
\hline \multirow{2}{*}{$\gamma$} & \multicolumn{3}{|c|}{$0.5 \%$ target loss } & \multicolumn{3}{c|}{$1 \%$ target loss } & \multicolumn{3}{c|}{$10 \%$ target loss } \\
\cline { 2 - 10 } & $L_{a}$ & $L_{s}$ & Error in $\%$ & $L_{a}$ & $L_{s}$ & Error in $\%$ & $L_{a}$ & $L_{s}$ & Error in \% \\
\hline 3.3 & 0.0645 & 0.0608 & 6.0 & 0.0757 & 0.0713 & 6.2 & 0.152 & 0.145 & 4.8 \\
4.0 & 0.0630 & 0.0645 & 2.4 & 0.0766 & 0.0791 & 3.2 & 0.169 & 0.164 & 3.0 \\
4.5 & 0.0628 & 0.0669 & 6.12 & 0.0773 & 0.080 & 3.4 & 0.177 & 0.168 & 5.3 \\
\hline
\end{tabular}

Similarily the fitted function for $B$ is as follows :

$$
B= \begin{cases}-0.012 \gamma^{3}-0.18 \gamma^{2}+0.905 \gamma-1.208, & \text { for slotted Aloha } \\ 0.0088 \gamma^{3}-0.139 \gamma^{2}+0.731 \gamma-0.974, & \text { for pa-Aloha } \\ 0.0061 \gamma^{3}-0.106 \gamma^{2}+0.613 \gamma-0.833 & \text { for pm-Aloha }\end{cases}
$$

Thus, the closed-form expression of packet loss rate for all $\gamma>2.0$, based on curve fitting techniques, is formed by (30), (33) and (34).

\section{Performance analysis with the 2 best receivers}

In this section, the effectiveness of proposed analytical model, based on curve fitting technique, is studied. The comparison between packet loss obtained from curve-fitting-based analytical model and R-SIM simulation results are shown in Fig. 3, Fig. 4 and Fig. 5 for S-Aloha, Pa-Aloha and Pm-Aloha, respectively.

In all the following figures, for two types of Aloha, we observe that the disparity between R-SIM simulation results and analytical model can be neglected, especially with the increase of the normalized load. To better illustrate the accuracy of the curve-fitting-based analytical model, we take Pa-Aloha as an example. Given a target packet loss rate and path loss exponent, the corresponding maximum supported normalized load is calculated. Three target packet loss rates, $0.5 \%, 1 \%, 10 \%$, are considered. The results are resumed into Tab. 2 : we observe that for all cases the errors between simulation results and analytical results are less than $10 \%$.

Now, we study the system capacity gain brought by MRC compared with SC. The system capacity is defined as the maximum load corresponding to a packet loss probability of $10 \%$. The system capacities of SC-based LPWAN system are obtained from realistic simulation. For MRC-based LPWAN system, the analytical model formed by (30), (32) and (33) can be used. We consider Pa-Aloha because this is most frequent scheme. As shown in Tab. 3, the system capacity is at least multiplied by a factor 1.26 when 2-best-RUs MRC is used instead of SC. The factor reaches 1.39 when $\gamma=3.3$, which confirms the interest of MRC macro diversity. Note that the analysis considers only 2 RUs in the MRC process. A larger capacity gain can be achieved if more RUs are involved into MRC.

\section{Conclusion}

In this paper, we proposed an analytical framework to evaluate the network packet loss rate of LPWAN systems applying MRC macro diversity. We obtained a closed-form expression based 

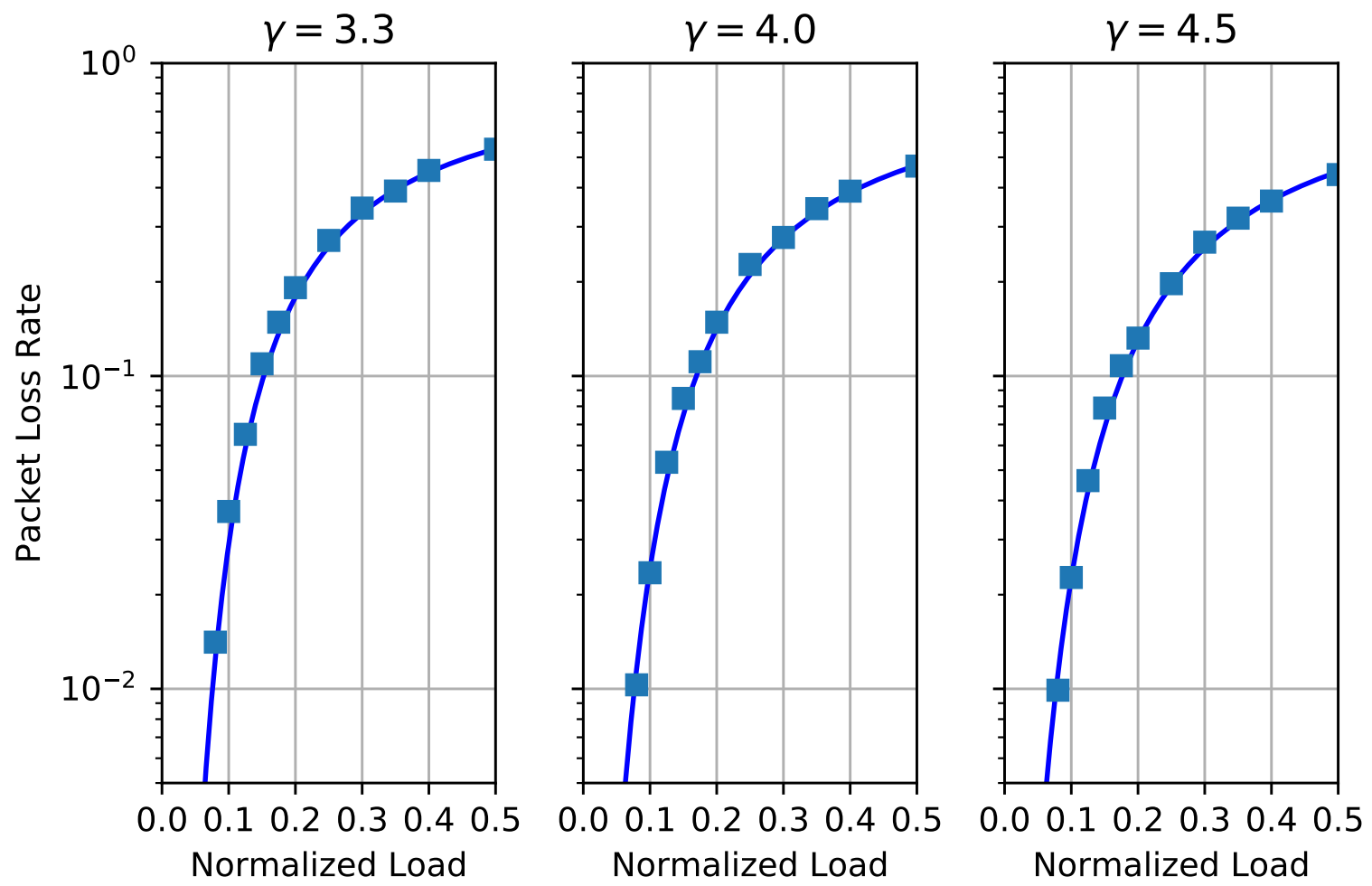

FiguRe 5 - Approximate closed-formula vs simulation for Pa-Aloha

TABle 3 - Maximum normalized load of Pa-Aloha with SC and MRC macro diversity for $10 \%$ loss probability

\begin{tabular}{|c|c|c|c|c|c|c|}
\hline \multirow{2}{*}{$\gamma$} & \multicolumn{3}{|c|}{$\theta_{\mathrm{dB}}=3 \mathrm{~dB}$} & \multicolumn{3}{c|}{$\theta_{\mathrm{dB}}=6 \mathrm{~dB}$} \\
\cline { 2 - 7 } & SC & MRC & Gain & SC & MRC & Gain \\
\hline 3.3 & 0.109 & 0.152 & $\times 1.39$ & 0.071 & 0.10 & $\times 1.40$ \\
\hline 4.0 & 0.127 & 0.169 & $\times 1.33$ & 0.088 & 0.12 & $\times 1.36$ \\
\hline 4.5 & 0.140 & 0.177 & $\times 1.26$ & 0.103 & 0.13 & $\times 1.26$ \\
\hline
\end{tabular}


on curve fitting technique for path loss exponent in range [3.3,4.5]. Our analysis covers both pure and slotted ALOHA. Through simulation, we confirmed the effectiveness of our proposed framework. We showed that MRC macro diversity can multiply the capacity by a factor greater than 1.26 if MRC leverages 2 nearest RUs. The capacity gain will be much higher if more RUs are involved.

\section{Conflict of Interest Statement}

The authors declare that they have no conflict of interest

\section{Références}

[1] Usman Raza, Parag Kulkarni, and Mahesh Sooriyabandara. Low power wide area networks : An overview. IEEE Communications Surveys \& Tutorials, 19(2) :855-873, 2017.

[2] Qipeng Song, Xavier Lagrange, and Loutfi Nuaymi. Evaluation of Macro Diversity Gain in Long Range ALOHA Networks. IEEE Communications Letters, 2017.

[3] Jahangir H Sarker and Hussein T Mouftah. Self-stability of slotted aloha by limiting the number of retransmission trials in infrastructure-less wireless networks. Telecommunication Systems, 52(2) :435-444, 2013.

[4] Majid Ghanbarinejad and Christian Schlegel. Distributed probabilistic medium access with multipacket reception and markovian traffic. Telecommunication Systems, 56(2) :311-321, 2014.

[5] Alessio Meloni and Maurizio Murroni. Interference calculation in asynchronous random access protocols using diversity. Telecommunication Systems, 63(1) :45-53, 2016.

[6] Peter Jung, Bernd Steiner, and Bernd Stilling. Exploitation of intracell macrodiversity in mobile radio systems by deployment of remote antennas. In Spread Spectrum Techniques and Applications Proceedings, 1996., IEEE 4th International Symposium on, volume 1, pages 302-307. IEEE, 1996.

[7] Marco Di Renzo, Fabio Graziosi, and Fortunato Santucci. A unified framework for performance analysis of CSI-assisted cooperative communications over fading channels. IEEE Transactions on Communications, 57(9) :2551-2557, 2009.

[8] Hesham ElSawy, Ekram Hossain, and Martin Haenggi. Stochastic geometry for modeling, analysis, and design of multi-tier and cognitive cellular wireless networks : A survey. IEEE Communications Surveys \& Tutorials, 15(3) :996-1019, 2013.

[9] Elvino S. Sousa and John A. Silvester. Optimum transmission ranges in a direct-sequence spread-spectrum multihop packet radio network. IEEE journal on selected areas in communications, 8(5) :762-771, 1990.

[10] Martin Haenggi. User point processes in cellular networks. IEEE Wireless Communications Letters, 6(2) :258-261, 2017. 
[11] Martin Haenggi. Diversity loss due to interference correlation. IEEE Communications Letters, 16(10):1600-1603, 2012.

[12] Ralph Tanbourgi, Harpreet S Dhillon, Jeffrey G Andrews, and Friedrich K Jondral. Effect of spatial interference correlation on the performance of maximum ratio combining. IEEE Transactions on Wireless Communications, 13(6) :3307-3316, 2014.

[13] Barłomiej Błaszczyszyn and Paul Mühlethaler. Interference and SINR coverage in spatial non-slotted Aloha networks. Annals of telecommunications, 70(7-8) :345-358, 2015.

[14] Harpreet S Dhillon and Jeffrey G Andrews. Downlink rate distribution in heterogeneous cellular networks under generalized cell selection. IEEE Wireless Communications Letters, $3(1): 42-45,2014$.

[15] Martin Haenggi and Radha Krishna Ganti. Interference in large wireless networks. Now Publishers Inc, 2009.

[16] John G. Proakis. Digital Communications 5th Edition. McGraw Hill, 2007.

[17] Stephen J Wolfe. On moments of probability distribution functions. In Fractional Calculus and Its Applications, pages 306-316. Springer, 1975.

[18] Ali Hirsa. Computational methods in finance. CRC Press, 2012.

[19] Qipeng Song, Xavier Lagrange, and Loutfi Nuaymi. An analytical model for S-ALOHA performance evaluation in M2M networks. In 2017 IEEE International Conference on Communications (ICC), pages 1-7. IEEE, 2017. 